University of Nebraska - Lincoln

DigitalCommons@University of Nebraska - Lincoln

Evaluation Of Single And Two-Stage Adaptive Sampling Designs For Estimation Of Density And Abundance Of Freshwater Mussels In A Large River

D. R. Smith

USGS, Leetown Science Center, drsmith@usgs.gov

J. T. Rogala

USGS, Upper Midwest Environmental Sciences Center, jrogala@usgs.gov

B. R. Gray

USGS, Upper Midwest Environmental Sciences Center, brgray@usgs.gov

Steven J. Zigler

USGS, szigler@usgs.gov

Teresa J. Newton

USGS, tnewton@usgs.gov

Follow this and additional works at: https://digitalcommons.unl.edu/usgsstaffpub

Smith, D. R.; Rogala, J. T.; Gray, B. R.; Zigler, Steven J.; and Newton, Teresa J., "Evaluation Of Single And Two-Stage Adaptive Sampling Designs For Estimation Of Density And Abundance Of Freshwater Mussels In A Large River" (2011). USGS Staff -- Published Research. 609.

https://digitalcommons.unl.edu/usgsstaffpub/609

This Article is brought to you for free and open access by the US Geological Survey at DigitalCommons@University of Nebraska - Lincoln. It has been accepted for inclusion in USGS Staff -- Published Research by an authorized administrator of DigitalCommons@University of Nebraska - Lincoln. 


\title{
EVALUATION OF SINGLE AND TWO-STAGE ADAPTIVE SAMPLING DESIGNS FOR ESTIMATION OF DENSITY AND ABUNDANCE OF FRESHWATER MUSSELS IN A LARGE RIVER $^{\dagger}$
}

\author{
D. R. SMITH, ${ }^{\mathrm{a} *}$ J. T. ROGALA, ${ }^{\mathrm{b}}$ B. R. GRAY, ${ }^{\mathrm{b}}$ S. J. ZIGLER ${ }^{\mathrm{b}}$ and T. J. NEWTON ${ }^{\mathrm{b}}$ \\ a USGS, Leetown Science Center, Kearneysville, WV 25430, USA \\ ${ }^{\mathrm{b}}$ USGS, Upper Midwest Environmental Sciences Center, La Crosse, WI 54603, USA
}

\begin{abstract}
Reliable estimates of abundance are needed to assess consequences of proposed habitat restoration and enhancement projects on freshwater mussels in the Upper Mississippi River (UMR). Although there is general guidance on sampling techniques for population assessment of freshwater mussels, the actual performance of sampling designs can depend critically on the population density and spatial distribution at the project site. To evaluate various sampling designs, we simulated sampling of populations, which varied in density and degree of spatial clustering. Because of logistics and costs of large river sampling and spatial clustering of freshwater mussels, we focused on adaptive and non-adaptive versions of single and two-stage sampling. The candidate designs performed similarly in terms of precision (CV) and probability of species detection for fixed sample size. Both CV and species detection were determined largely by density, spatial distribution and sample size. However, designs did differ in the rate that occupied quadrats were encountered. Occupied units had a higher probability of selection using adaptive designs than conventional designs. We used two measures of cost: sample size (i.e. number of quadrats) and distance travelled between the quadrats. Adaptive and two-stage designs tended to reduce distance between sampling units, and thus performed better when distance travelled was considered. Based on the comparisons, we provide general recommendations on the sampling designs for the freshwater mussels in the UMR, and presumably other large rivers. Published in 2009 by John Wiley \& Sons, Ltd.
\end{abstract}

KEY WORDS: benthic sampling; adaptive cluster sampling; two-stage sequential sampling; two-stage sampling; unionids; freshwater mussels

Received 19 December 2008; Revised 15 October 2009; Accepted 20 October 2009

\section{INTRODUCTION}

Freshwater mussels (Unionoidea) are among the most imperilled of all taxonomic groups (Ricciardi and Rasmussen, 1999; Strayer et al., 2004). The Upper Mississippi River (UMR) supports a diverse and valuable freshwater mussel assemblage, and conservation of native mussels is of great importance to resource managers (UMRCC, 1990). Considerable effort is being expended to predict the effects of proposed habitat rehabilitation efforts on mussels, but estimates of effects can range widely. For example, estimates of Unionid mortality in 2005 as a result of the drawdown of Pool 5 range from about 100000 to upwards of several million (Wisconsin DNR unpublished data). Reliable estimates of abundance are needed to rigorously assess consequences of proposed habitat restoration and enhancement projects on mussels.

Although there is general guidance on sampling techniques for population assessment of freshwater mussels

\footnotetext{
*Correspondence to: D. R. Smith, USGS, Leetown Science Center, Aquatic Ecology Lab, 11649 Leetown Road, Kearneysville, WV 25430, USA. E-mail: drsmith@usgs.gov

${ }^{\dagger}$ This paper is a US Government work and is in the public domain in the USA.
}

(Strayer and Smith, 2003), the performance of sampling designs can depend critically on the spatial distribution, density and characteristics of the target population and study site. It is impractical, if not impossible, to evaluate a wide range of sampling designs through field trials to determine how well density can be estimated. However, computer simulations can be useful for evaluating sampling designs and narrowing the field of potentially efficient designs (Christman, 2000; Morrison et al., 2008). Thus, we used computer simulations that incorporated observed densities and spatial clustering from mussel surveys in the UMR to evaluate various sampling designs.

There are a number of sampling designs that warrant consideration because of the spatial distribution of freshwater mussels and the logistic challenges inherent to sampling large rivers. Adaptive sampling designs are a class of probabilistic sampling designs that are suitable for sampling populations that are spatially rare and clustered, such as freshwater mussels (Downing and Downing, 1992). Adaptive designs allow sampling effort to change (usually increase) in response to what is found while sampling and provide design-unbiased estimates of population totals and means (Thompson, 2002; Brown, 2003). For clustered populations, such as freshwater mussel populations, more 
individuals and species will be collected with adaptive sampling compared to conventional probabilistic designs for fixed sampling effort (Smith et al., 2003). For clustered and spatially rare populations, adaptive sampling designs might result in lower variance compared to conventional probabilistic designs for fixed sampling effort (Brown, 2003; Smith et al., 2004).

When sampling large rivers, such as the UMR, it is often logistically efficient to sample in two-stages by first selecting several areas within the river to work, and then subsampling those areas. In survey parlance, the areas are called 'primary units' and subsampling units are called 'secondary units'. Two-stage designs can be used to reduce movement within large rivers, which can be expensive, timeconsuming and potentially dangerous. There are adaptive and non-adaptive versions of two-stage sampling (Salehi and Seber, 1997). Because adaptively sampled units tend to be clustered, adaptive sampling can also help to reduce distance between sampled units when applied within either single or two-stage designs.

Two-stage sequential sampling incorporates elements of adaptive sampling into a two-stage design (Salehi and Smith, 2005). In this design, subsampling occurs sequentially, but only within those areas that meet a criterion associated with occurrence or density of the target species. The criterion is based on the results of an initial subsample that is taken within all areas. If the criterion is met within an area, then a sequential subsample is taken.

In this paper we evaluate several sampling designs that are potentially useful for estimation of density and abundance of freshwater mussels in the UMR. We focus on comparing adaptive and non-adaptive versions of single and two-stage sampling. We compare the precision and effectiveness of the designs on populations, which vary in density and degree of spatial clustering. Because encountering rare species is often a goal in mussel surveys, we also compare encounter rates among designs. Based on the comparisons, we provide general recommendations on the best sampling designs.

\section{METHODS}

\section{Population generation}

We generated populations that ranged in density and spatial clustering. Each population is a realization of a spatial point process (Diggle, 1983). The populations were simulated to approximate density and spatial clustering of mussel species found within a 60 ha study area defined by the East Channel, Pool 10. The sample data from Pool 10 (Holland-Bartels, 1990) were summarized to provide a basis for generating population data. The variance to mean ratio, an indicator of spatial clustering, ranged from 4 to 7 with most between 6 and 6.5. Simulated densities were 0.001, $0.01,0.05,0.20,0.40$ and $1.5 \mathrm{~m}^{-2}$. These densities are generally representative of threatened and endangered species in the UMR, and $75 \%$ of species-specific densities from Holland-Bartels (1990) were $<1.5 \mathrm{~m}^{-2}$.

To generate spatial patterns, two spatial-point processes were used, either Poisson cluster process (Brown, 2003) for a distribution of tightly clustered populations or Matern process (Petitgas, 2003) for loose elliptical clusters. Predicted spatial distributions of freshwater mussels in the Allegheny River from kriging of sample data resemble a Poisson cluster process (Smith et al., 2001a). In the Mississippi River, mussels are expected to distribute within 'beds' shaped by the current (Morales et al., 2006), and the Matern process is meant to approximate that spatial distribution.

Spatial pattern of populations following the Poisson cluster process are generated in two-stages (Brown, 2003). First, the number of clusters was selected from a Poisson distribution, and cluster centres were randomly located throughout the site. Second, individuals within the cluster were located around the cluster centre at a random distance following an exponential distribution and a random direction following a uniform distribution. Spatial pattern of the populations following the Matern process was generated in a similar way to the Poisson cluster process except that: (1) the number of clusters was not predetermined, rather clusters and individuals were generated until the target abundance was met and (2) the cluster shape was an ellipse within which individuals were located randomly. The long-axis of the ellipse was oriented in a random direction within 10 degrees of the dominant thalweg. Cross-channel gradients were also incorporated, which resulted in three patterns: Poisson clusters with and without a gradient and the Matern clusters with a gradient. The populations are shown in Figures 1-3.

\section{Sampling designs and simulation}

For each population, sampling was simulated using the following five designs. Additional details on the sampling designs can be found in Thompson and Seber (1996), Salehi and Seber (1997) and Salehi and Smith (2003).

(1) Single-stage conventional simple random sampling (SRS): this was the 'null' sampling model used for comparison with other approaches. Sample size was the only design factor.

(2) Single-stage adaptive simple random sampling (ASRS): this was an adaptive cluster sampling approach with the initial sample plot selected by simple random sampling. The design factors were initial sample size, condition to adapt and neighbourhood. The condition to adapt was the count within a sampling unit; this triggered adaptive sampling in the neighbourhood of the sampling unit. The neighbourhood was defined as all units that shared a 


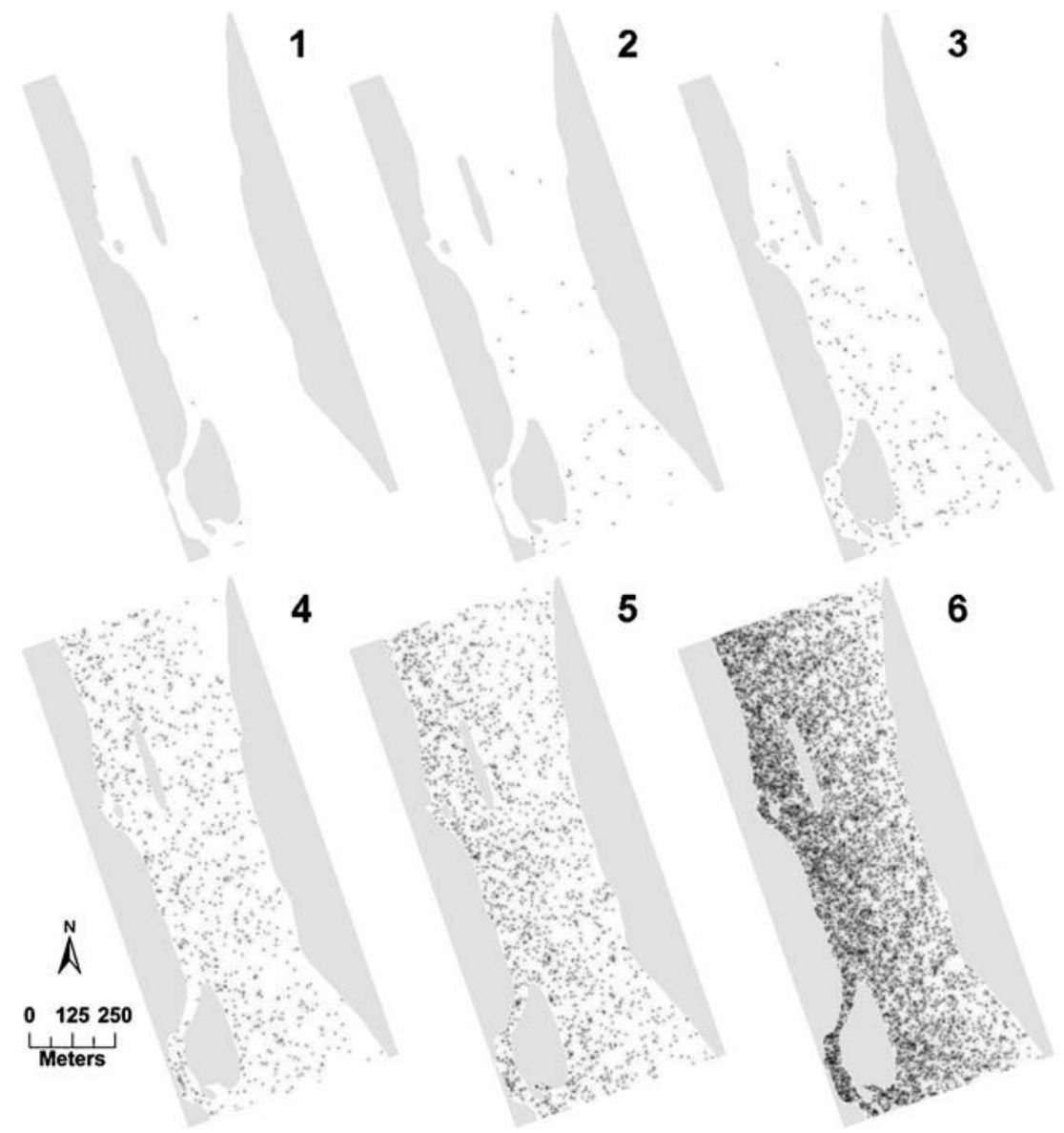

Figure 1. Populations 1-6 follow a Poisson clustering process with a cross-river gradient. (Populations generated to evaluate sampling designs). The Poisson cluster process results in a tightly clustered spatial distribution, and the Matern process results in a loosely clustered spatial distribution. In all figures, densities were $0.001,0.01,0.05,0.20,0.40$ and $1.5 \mathrm{~m}^{-2}$ corresponding to the increasing population numbers

side with the unit that met the condition to adapt; this neighbourhood definition was used in all adaptive designs. The modified Horvitz-Thompson estimator was used to estimate density (Thompson and Seber, 1996).

(3) Two-stage conventional simple random sampling (TS SRS): in this design, the river was divided into large (primary) units, e.g. $80 \mathrm{~m}$ by $80 \mathrm{~m}$. Then sampling proceeded in stages. First, we selected a sample of primary units. Second, within selected primary units, we selected a sample of quadrats (i.e. secondary units). The design factors included the size (dimensions) of the primary units, the sample size of primary units, and the sample size of quadrats within each primary unit. For convenience, we also referred to the sample size of primary units as the fraction of primary units that were selected (i.e. $f_{\mathrm{psu}}$ ); the sample size of primary units $=$ the fraction selected $\times$ the total number of primary units in the population.
(4) Two-stage adaptive simple random sampling (TS ASRS): this was a combination of ASRS and TS SRS. In this design, the river was divided into primary units, which were selected randomly at the first stage. Then, within each primary unit adaptive sampling (ASRS) was applied at the second (quadrat-level) stage. Design factors for both ASRS and TS SRS were included.

(5) Two-stage sequential sampling (TS SS): like TS SRS, the river was divided into primary units, which were selected randomly at the first stage. At the second stage, an initial random sample of quadrats was taken within a primary unit, and then if a condition was met on any of those quadrats, a sequential pre-defined sample of quadrats was taken. Thus, this design was also an adaptive design in that sampling effort increased if freshwater mussels were encountered. However, the increased sampling effort was not restricted to a neighbourhood, but was distributed throughout the primary unit. The design factors were the size (dimensions) of the primary 


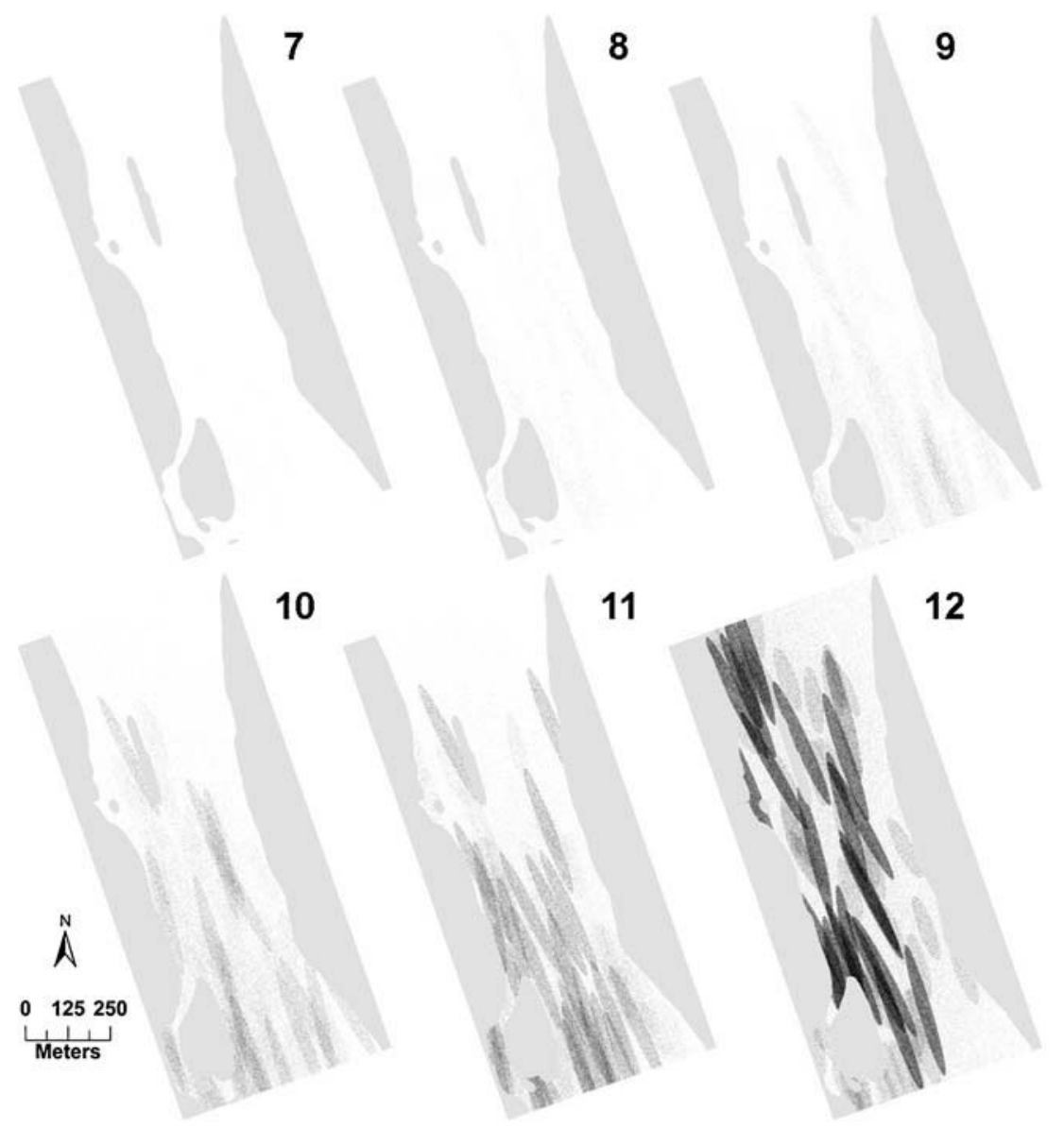

Figure 2. Populations 7-12 follow a Matern process with a cross-river gradient. (Populations generated to evaluate sampling designs). The Poisson cluster process results in a tightly clustered spatial distribution, and the Matern process results in a loosely clustered spatial distribution. In all figures, densities were $0.001,0.01,0.05,0.20,0.40$ and $1.5 \mathrm{~m}^{-2}$ corresponding to the increasing population numbers

units, the sample size of primary units (or fraction selected, i.e. $f_{\mathrm{psu}}$ ), the sample size for the initial sample of quadrats, and the sample size for the sequential sample of quadrats.

The condition for adaptive (ASRS, TS ARS) and sequential (TS SS) sampling was a count $>0$. Quadrat area was the nominal standard $0.25 \mathrm{~m}^{2}$ for all designs. Adaptive sampling was not simulated for the highest density (i.e. $1.5 \mathrm{~m}^{-2}$ ) because we anticipated that in high-density populations, the final sample size would be much higher than the initial sample size, which leads to inefficient designs (Smith et al., 2004). For the two-stage designs, the study area was divided into 10,20 and 55 primary units, representing areas of approximately 6,3 and 1.1 ha, respectively. Primary units were created by overlaying a grid on the rectangle that encompasses the river reach. Primary units were unequal in size because of the irregular shape of the river. Estimators for two-stage designs allow for unequal sized primary units. Results were similar among levels within this design factor. So, we present only the results from the division into 20 primary units.

We used a simulation program SAMPLE that was designed for simulating and comparing sampling designs, particularly adaptive designs (Morrison et al., 2008). Each design was simulated 1000 times. The software can be downloaded at http://www.lsc.usgs.gov/aeb/davids/acs/.

\section{Comparison of design performance}

To evaluate design performance, we compared coefficient of variation $(\mathrm{CV})$, probability of detecting a species and the relative risk of sampling an occupied quadrat. $\mathrm{CV}$ is the ratio of standard error (SE) over density, where SE is the standard deviation of the density estimate. Probability of species detection is the probability of sampling at least one freshwater mussel during a survey. Relative risk is the ratio of the proportion of occupied quadrats in the sample $\left(p_{1}\right)$ relative to that of the population $\left(p_{2}\right)$. Thus, relative risk of sampling an occupied quadrat $=p_{1} / p_{2}$. The success of 




Figure 3. Close up view of populations 2, 3, 8 and 9. Populations 2 and 3 are tightly clustered (Poisson cluster process) and have densities of 0.01 and $0.05 \mathrm{~m}^{-2}$, respectively. Populations 8 and 9 are loosely clustered (Matern process) and have densities of 0.01 and $0.05 \mathrm{~m}^{-2}$, respectively

encountering occupied units can also be measured using odds ratios (Agresti, 1990),

$$
\text { Odds ratio }=\text { Relative risk } \times \frac{\left(1-p_{2}\right)}{\left(1-p_{1}\right)}
$$

Comparison of design performance must take into account variation in sampling effort and cost. Thus, we compared CV, probability of species detection and proportion of occupied sample quadrats $\left(p_{1}\right)$ among designs for given sampling effort and cost. Sampling effort and cost can be measured by sample size or a surrogate measure of cost, such as distance travelled. Reducing distance travelled can be important in surveys of large rivers and useful for comparing two-stage and adaptive designs. Expected sample size, as measured by the average across replications, was used in these comparisons for adaptive and sequential designs.

We used general linear models to compare CV among designs while controlling for population density, sampling effort (sample size) and spatial clustering. We had two objectives in our modelling of CV. One objective was descriptive-to describe the relationships between precision and characteristics of the design and population. We found that an ANOVA model with transformed sample size and separate analysis for each density to be a straightforward approach for this objective. Sample size was log transformed to improve fit and meet linear model assumptions. The other objective was predictive- to predict the expected precision based on characteristics of the design and populations. We found it most useful to predict on the original scale and incorporate density as a predictor. So, a nonlinear 
model (power function) was the better choice for this objective.

We used logistic regression to model the probability of species detection. The probability of species detection was calculated as the frequency that at least one occupied quadrat was selected in a sample among all replicated samples. For densities $\geq 0.2 \mathrm{~m}^{-2}$, the probability of species detection was high across all sample sizes. Thus, we limited the modelling of species detection to populations with densities of $0.001,0.01$ and $0.05 \mathrm{~m}^{-2}$, and we fit the logistic model separately for each density as we did with the ANOVA model. The logistic model was (Hosmer and Lemeshow, 1989)

$$
\begin{aligned}
\ln \left(\frac{\pi(x)}{1-\pi(x)}\right) \\
=\beta_{0}+\sum_{u=1}^{4} \beta_{1 u} D_{1 u}+\beta_{2} D_{2}+\beta_{3} v+\sum_{u=1}^{4} \beta_{4 u} D_{1 u} D_{2} \\
\quad+\sum_{u=1}^{4} \beta_{5 u} D_{1 u} v+\beta_{6} D_{2} v
\end{aligned}
$$

where $\pi(x)$ denotes the frequency of species detection given covariates, and the covariates were denoted by $D_{1}$ for design, simulations to low densities (i.e. 0.001 and $0.01 \mathrm{~m}^{-2}$ ) and 500 replications.

\section{RESULTS}

The presence of a cross-channel gradient did not affect the results for the Poisson cluster populations. Thus, we report comparisons among the populations that included a crosschannel gradient, i.e. the tightly clustered (Poisson cluster process) populations and the loosely clustered (Matern process) populations (Figures 1 and 2).

Coefficient of variation was a function of density, spatial distribution, design, sample size and interactions (Table I). $R^{2}$ estimates were highest (i.e. 0.97 ) for density $=0.01 \mathrm{~m}^{-2}$. As density increased, the CV model was less predictive and only sample size and design were significant predictors. The model was also less predictive for density $0.001 \mathrm{~m}^{-2}$. However, CVs differed little among designs, being largely determined by spatial distribution, density and sample size (Table I, Figure 4). Coefficient of variation tended to be somewhat higher for the Poisson cluster process.

A nonlinear (power function) model was used to predict $\mathrm{CV}$ as a function of spatial distribution, density and sample size (Figure 5). The model was fit separately for each spatial distribution. The resulting model equations were

$$
\mathrm{CV}=\left\{\begin{array}{l}
268 \mu^{-0.50} v^{-0.51} \text { for Poisson cluster, tightly clustered population } \\
250 \mu^{-0.47} v^{-0.50} \text { for Matern cluster, loosely clustered population }
\end{array}\right.
$$

$D_{2}$ for spatial distribution and $v$ for expected sample size. Regression models (glm and logistic) were fit using SAS.

\section{Comparison of distance travelled among designs}

Much of the cost of sampling in large rivers results from travel among sampling units (in this case, among quadrats) and costs associated with positioning the boat and divers. These costs will be proportional to cumulative distance travelled because units that are close can be sampled from the same position. This is especially true for adaptive networks. We calculated distance travelled to compare design performance for fixed cost. Distance travelled $(d)$ was calculated by starting at the southwest corner of the site and computing the distance $\left(d_{1}\right)$ to the nearest unit (quadrat or network). Then from the first unit, the distance $\left(d_{2}\right)$ to the nearest unit was calculated. This was continued for the remainder of the sample, and distance travelled was the sum of the unit-to-unit distances, i.e. $d=\sum_{i=1}^{v} d_{i}$. Distance was calculated for each replication and then averaged to generate an expected distance travelled for a given design. Because of the time required to compute distance, we limited the where $\mu$ is density $\left(\mathrm{m}^{-2}\right)$ and $v$ is sample size.

The probability that a species was detected was determined largely by population density and sample size, and the probability varied little among designs (Figure 6). For all designs, species detection exceeded $90 \%$ for sample sizes $\geq 400$ and densities $\geq 0.05 \mathrm{~m}^{-2}$. For densities of 0.4 and $1.5 \mathrm{~m}^{-2}$, probability of detection exceeded 0.99 across the full range of sample sizes. However, for densities of $0.01 \mathrm{~m}^{2}$, probability of species detection did not exceed 0.9 until sample size exceeded 1000 regardless of design.

Spatial distribution and design were the most important factors in predicting the rate that occupied quadrats were encountered (Table II). The logistic regression models of encountering occupied quadrats as a function of spatial distribution, design, sample size and interactions were highly predictive across the range of densities. $R^{2}$ estimates exceeded 0.9 except for density of $1.5 \mathrm{~m}^{-2}$.

Adaptive designs increased the rate that occupied quadrats were encountered by a factor of 3-6 over conventional designs for tightly clustered (Poisson cluster) populations (Figure 7). The odds ratios for adaptive designs were significant for densities $\geq 0.01$ in tightly clustered 
Table I. ANOVA results for model of sample CV as a function of spatial distribution, design, sample size and interactions. Spatial distributions were tightly clustered, based on Poisson cluster process, or loosely clustered, based on Matern process. Designs were conventional and adaptive simple random sampling, two-stage conventional and adaptive sampling and two-stage sequential sampling. Final sample sizes ranged from 100 to over 2000 quadrats. Sample size was log transformed. The model was fit separately for each population density. No other interactions were significant except for those shown

\begin{tabular}{|c|c|c|c|c|}
\hline Density $\left(\mathrm{m}^{-2}\right)$ & $R^{2}$ & Effect & $F$ & $p$ \\
\hline \multirow[t]{4}{*}{0.001} & \multirow[t]{4}{*}{0.88} & Spatial distribution & 49.74 & $<0.0001$ \\
\hline & & Design & 13.51 & $<0.0001$ \\
\hline & & Sample size & 624.78 & $<0.0001$ \\
\hline & & Design $\times$ Sample size & 2.46 & 0.0500 \\
\hline \multirow[t]{4}{*}{0.01} & \multirow[t]{4}{*}{0.97} & Spatial distribution & 162.94 & $<0.0001$ \\
\hline & & Design & 44.17 & $<0.0001$ \\
\hline & & Sample size & 2747.91 & $<0.0001$ \\
\hline & & Design $\times$ Sample size & 2.38 & 0.0567 \\
\hline \multirow[t]{4}{*}{0.05} & \multirow[t]{4}{*}{0.95} & Spatial distribution & 63.45 & $<0.0001$ \\
\hline & & Design & 16.26 & $<0.0001$ \\
\hline & & Sample size & 1644.28 & $<0.0001$ \\
\hline & & Design $\times$ Sample size & 2.58 & 0.0418 \\
\hline \multirow[t]{4}{*}{0.20} & \multirow[t]{4}{*}{0.86} & Spatial distribution & 2.83 & 0.0956 \\
\hline & & Design & 2.41 & 0.0544 \\
\hline & & Sample size & 570.26 & $<0.0001$ \\
\hline & & Design $\times$ Sample size & 0.35 & 0.8418 \\
\hline \multirow[t]{4}{*}{0.40} & \multirow[t]{4}{*}{0.75} & Spatial distribution & 0.43 & 0.5152 \\
\hline & & Design & 1.88 & 0.1202 \\
\hline & & Sample size & 279.36 & $<0.0001$ \\
\hline & & Design $\times$ Sample size & 0.06 & 0.9922 \\
\hline \multirow[t]{4}{*}{1.50} & \multirow[t]{4}{*}{0.53} & Spatial distribution & 1.14 & 0.2901 \\
\hline & & Design & 4.46 & 0.0150 \\
\hline & & Sample size & 70.81 & $<0.0001$ \\
\hline & & Design $\times$ Sample size & 0.11 & 0.8916 \\
\hline
\end{tabular}

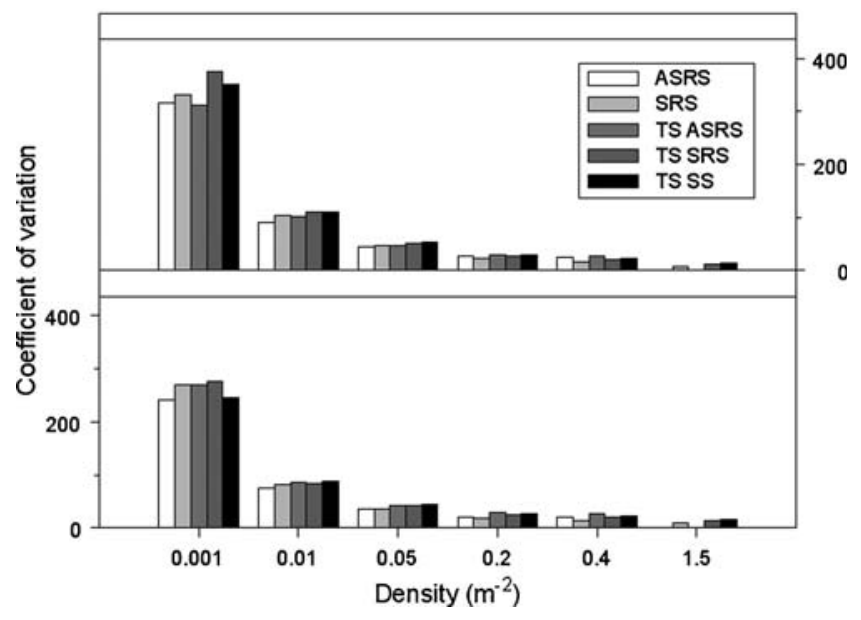

Figure 4. Coefficient of variation for five sampling designs, six densities and two spatial distributions. The top panel labelled Poisson shows results for populations with tightly clustered distributions (see Populations 1-6 in Figure 1). The bottom panel labelled Matern shows results for populations with loosely clustered distributions (see Populations $7-12$ in Figure 2). The sample size was 600 for all designs and populations shown
(Poisson cluster) populations and for densities $\geq 0.20$ in loosely clustered (Matern) populations (Figure 8).

Precision varied among designs when sampling cost was measured by the distance travelled (Figure 9). A model including factors of spatial distribution, density, design and distance travelled explained much of the variation in $\mathrm{CV}$ $\left(R^{2}=0.92\right)$. In three of the four cases applied to loosely clustered (Matern) populations, two-stage sampling with $40 \%$ of primary units selected (i.e. $f_{\mathrm{psu}}=0.4$ ) resulted in lower CV than other two-stage or single-stage designs for the full range of sampling effort (Figure 9). In other cases, $\mathrm{CV}$ was lowest for the $f_{\mathrm{psu}}=0.4$ case for at least part of the range of sampling effort.

\section{DISCUSSION}

The candidate designs performed similarly in terms of precision (CV) and probability of species detection for fixed sample size. Both CV and species detection were determined largely by density and sample size. However, designs differed in the rate that occupied quadrats were encountered. Occupied units had a higher probability of selection using adaptive designs than conventional designs. 




Figure 5. Predicted coefficient of variation $(\mathrm{CV})$ as a function of spatial distribution, density and sample size. Sampling design was not included as a factor in the model to generalize the prediction. Results from populations with tightly clustered distributions (Poisson cluster process) are shown in the top plot, and those with loosely clustered distributions (Matern process) are shown in the bottom plot. Model equations are shown in Equation 3

At times it is desirable in freshwater mussel surveys to encounter more occupied units so that more mussels can be collected and measured. This would be true when the analyses of demographics or habitat relationships are survey objectives (Strayer et al., 2004). In such cases, adaptive designs should be considered. The use and application of adaptive designs have many logistical considerations (Smith et al., 2004). Thus, prior to large-scale implementation, field trials should be conducted to work out how adaptive sampling could be conducted. Many modifications, such as use of stopping rules, are available to adapt the sampling protocol to logistic constraints (Brown and Manly, 1998; Brown, 2003).

The comparison of sampling design performance for fixed cost can be affected by how sampling cost is measured. We
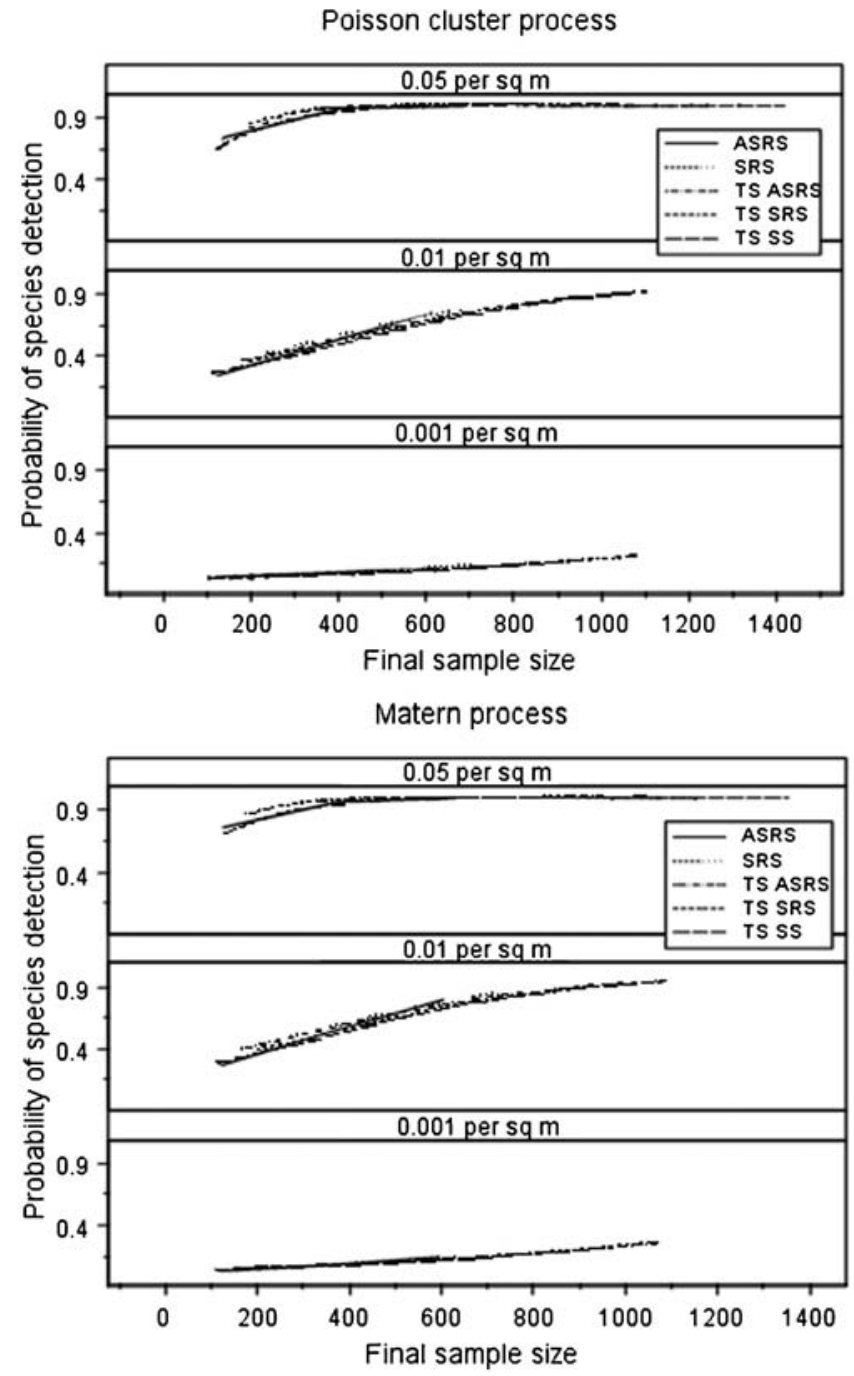

Figure 6. Probability of species detection predicted from logistic regressions as a function of spatial distribution, design, density and sample size. Results from populations with tightly clustered distributions (Poisson cluster process) are shown in the top plot, and those with loosely clustered distributions (Matern process) are shown in the bottom plot. Densities of 0.4 and $1.5 \mathrm{~m}^{-2}$ are not shown because probability of detection was high for the full range of sample size

used two measures of cost: sample size (i.e. number of quadrats) and distance travelled between the quadrats. Adaptive and two-stage designs tended to reduce distance between sampling units, and thus performed better when sampling cost was measured by distance travelled. Twostage designs with a moderate fraction of primary units selected (i.e. $f_{\mathrm{psu}}=0.4$ ) tended to yield more precise estimates for fixed distance travelled than single-stage designs or two-stage designs with a higher $f_{\mathrm{psu}}$. The combination of two-stage and adaptive designs yielded the most precise estimate for fixed distance travelled. 
Table II. ANOVA results from logistic regression model of the relative frequency of species detection as a function of spatial distribution, design, sample size and interactions. Spatial distributions were tightly clustered, based on Poisson cluster process, or loosely clustered, based on Matern process. Designs were conventional and adaptive simple random sampling, two-stage conventional and adaptive sampling and twostage sequential sampling. Final sample sizes ranged from 100 to over 2000 quadrats. The model was fit separately for each population density. The relative frequency of species detection is the proportion of sampled quadrats that are occupied by at least one freshwater mussel

\begin{tabular}{|c|c|c|c|c|}
\hline Density $\left(\mathrm{m}^{-2}\right)$ & $R^{2}$ & Effect & $F$ & $p$ \\
\hline \multirow[t]{5}{*}{0.001} & \multirow[t]{5}{*}{0.92} & Spatial distribution & 129.83 & $<0.0001$ \\
\hline & & Design & 18.10 & $<0.0001$ \\
\hline & & Sample size & 2.82 & 0.0961 \\
\hline & & Spatial distribution $\times$ Design & 80.89 & $<0.0001$ \\
\hline & & Design $\times$ Sample size & 1.14 & 0.3436 \\
\hline \multirow[t]{5}{*}{0.01} & \multirow[t]{5}{*}{0.99} & Spatial distribution & 1247.50 & $<0.0001$ \\
\hline & & Design & 176.58 & $<0.0001$ \\
\hline & & Sample size & 28.57 & $<0.0001$ \\
\hline & & Spatial distribution $\times$ Design & 778.94 & $<0.0001$ \\
\hline & & Design $\times$ Sample size & 9.02 & 0.0567 \\
\hline \multirow[t]{5}{*}{0.05} & \multirow[t]{5}{*}{0.99} & Spatial distribution & 1325.51 & $<0.0001$ \\
\hline & & Design & 194.66 & $<0.0001$ \\
\hline & & Sample size & 24.15 & $<0.0001$ \\
\hline & & Spatial distribution $\times$ Design & 914.73 & $<0.0001$ \\
\hline & & Design $\times$ Sample size & 11.29 & $<0.0001$ \\
\hline \multirow[t]{5}{*}{0.20} & \multirow[t]{5}{*}{0.99} & Spatial distribution & 2167.46 & $<0.0001$ \\
\hline & & Design & 662.62 & $<0.0001$ \\
\hline & & Sample size & 19.76 & $<0.0001$ \\
\hline & & Spatial distribution $\times$ Design & 1658.25 & $<0.0001$ \\
\hline & & Design $\times$ Sample size & 15.90 & $<0.0001$ \\
\hline \multirow[t]{5}{*}{0.40} & \multirow[t]{5}{*}{0.99} & Spatial distribution & 435.50 & $<0.0001$ \\
\hline & & Design & 572.62 & $<0.0001$ \\
\hline & & Sample size & 3.51 & 0.0639 \\
\hline & & Spatial distribution $\times$ Design & 403.60 & $<0.0001$ \\
\hline & & Design $\times$ Sample size & 6.39 & 0.0001 \\
\hline \multirow[t]{5}{*}{1.50} & \multirow[t]{5}{*}{0.73} & Spatial distribution & 135.73 & $<0.0001$ \\
\hline & & Design & 6.94 & 0.0017 \\
\hline & & Sample size & 0.08 & 0.7803 \\
\hline & & Spatial distribution $\times$ Design & 9.22 & 0.0003 \\
\hline & & Design $\times$ Sample size & 0.25 & 0.7765 \\
\hline
\end{tabular}

We did not evaluate systematic design here because it has been previously shown that systematic designs perform at least as well as conventional simple random sampling, especially for clustered populations (Christman, 2000; Pooler and Smith, 2005). Instead we focused on comparing adaptive and conventional sampling within single and twostage designs. That general comparison is likely to be consistent for systematic as well as simple random sampling. Systematic sampling is logistically feasible and provides good spatial balance. Systematic sampling can be combined with adaptive sampling by taking the initial sample systematically (Morrison et al., 2008).

Stratification can also be incorporated into sampling designs to allocate sampling effort to high-density areas (Thompson, 2002). If there is a known habitat association, then sampling could be stratified by habitat. Depth and flow would be useful habitat variables to consider. Two-phase sampling or double-sampling for stratification can be used in cases where a priori stratification variables are not available. Two-phase sampling combines qualitative and quantitative sampling methods and has been applied to surveys of freshwater mussels (Villella and Smith, 2005). Model-based designs could also be considered as a method to include a habitat association (Dorazio, 1999). Inference from modelbased designs relies on the realism of the underlying model (Thompson, 2002).

We focus on comparing precision among sampling designs. The sampling designs considered here are design-unbiased meaning that the expected value of the sample estimates equals the population parameter of interest (Thompson, 2002). For example, estimates of density from an adaptive sampling design, on average, equals population 
Poisson cluster process

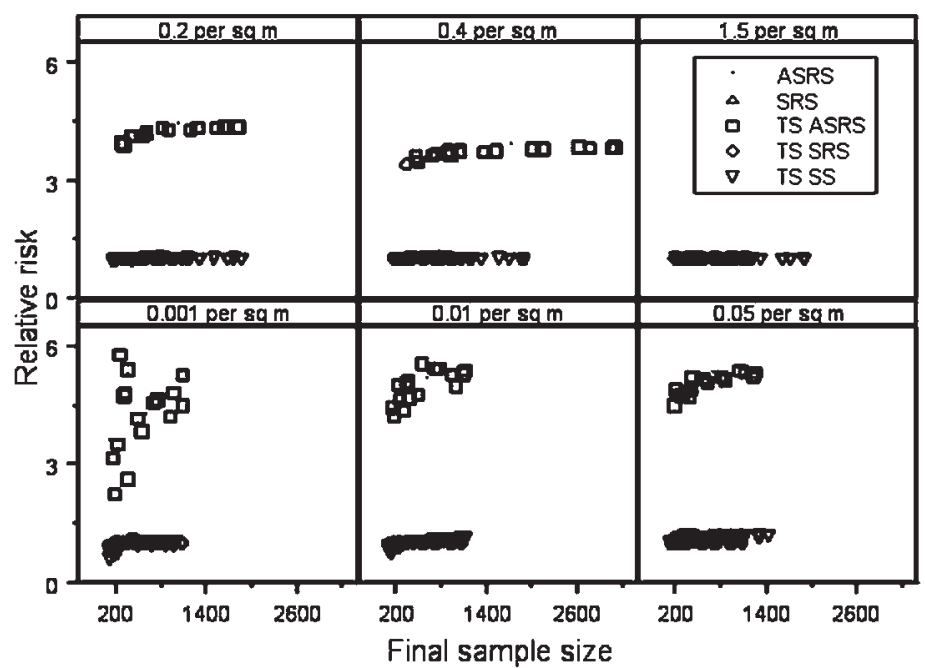

Matern process

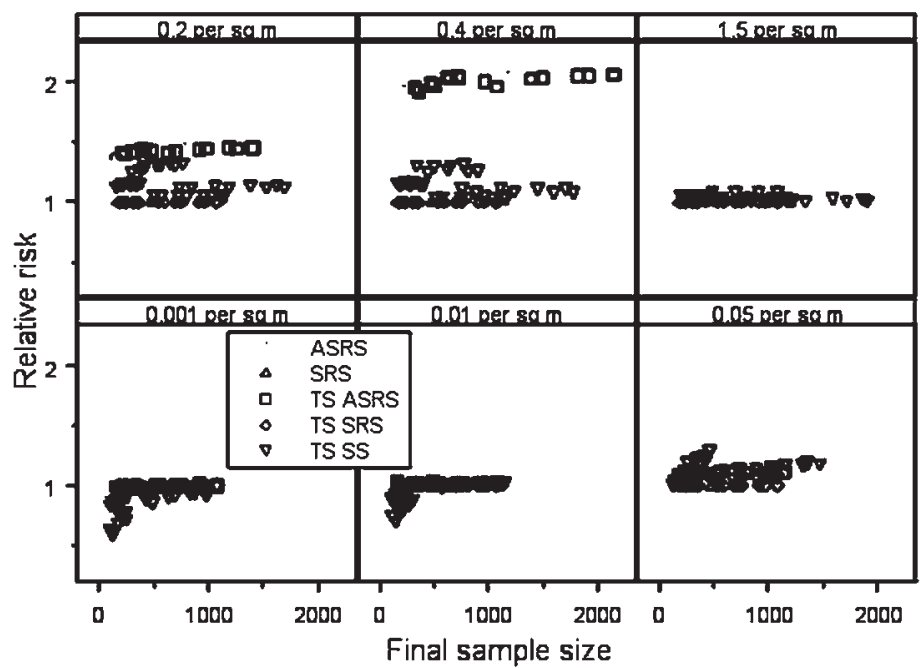

Figure 7. Relative risk of sampling an occupied quadrat as a function of spatial distribution, density and sampling design. Relative risk is the ratio of the expected proportion of the sampled quadrats that are occupied over the proportion of the population of quadrats that are occupied. Designs with relative risk $>1$ tend to encounter occupied units more frequently that at random. Populations with tightly clustered distributions (Poisson cluster process) are shown in the top

six panels, and those with loosely clustered distributions (Matern process) are shown in the bottom six panels

density. There are issues with bias caused by imperfect detection (i.e. detectability), which are relevant to any sampling design-probabilistic or otherwise. Imperfect detection is a form of measurement error. For example, mussels below the substrate surface, small mussels in particular, might not be detected even though the individual's location was randomly selected and searched (Smith et al., 2001b). Because quadrats are routinely excavated in surveys in large rivers, we assume that detectability is high except for small-sized or young mussels. The effect of detectability on estimates of abundance, particularly in adaptive designs, needs more research (Thompson and Seber, 1994).

Because precision of density or abundance estimates are determined largely by density and spatial distribution of the target species and sample size for a wide range of sampling designs, the choice of sampling design will depend on logistical considerations and alternative survey objectives, such as the desire to encounter occupied units. Logistical considerations tend to favour systematic and two-stage designs. The objective of encountering occupied units tends to favour adaptive and stratified designs. Designs can be 


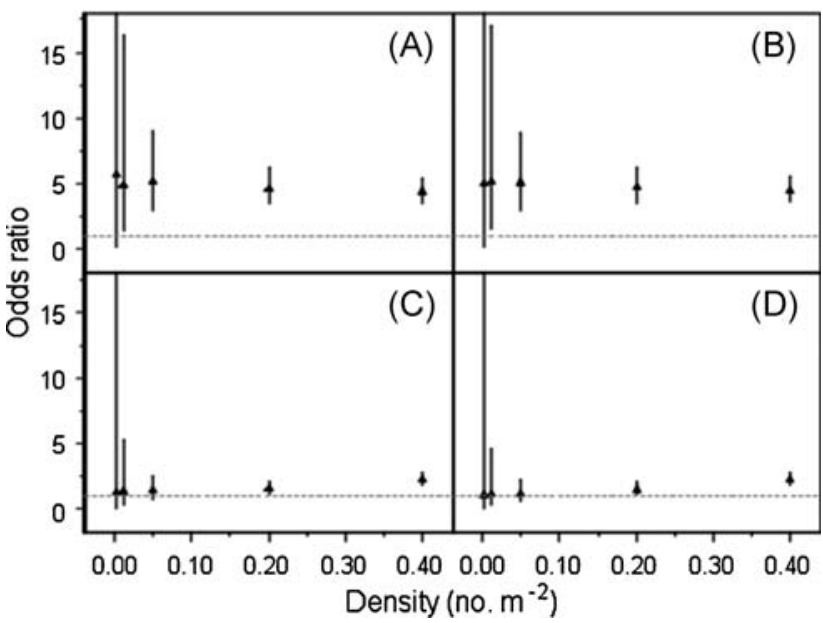

Figure 8. Odds ratios with $90 \%$ confidence intervals for encountering occupied units using adaptive versus conventional sampling designs (single-stage in panels A and C; two-stage in panels B and D). Results for populations with tightly clustered spatial distributions (Poisson cluster process) are shown in panels A and B. Results for populations with loosely clustered spatial distributions (Matern process) are shown in panels $C$ and $D$

combined, e.g. two-stage adaptive sampling, to satisfy logistical considerations and multiple survey objectives.

\section{CONCLUSIONS AND RECOMMENDATIONS}

- Reliability of estimates of freshwater mussel density or abundance in the UMR will be determined largely by population density and spatial distribution. For planning purposes, the following equations (derived from Equation 3) can be used to find approximate sample size to achieve a desired $\mathrm{CV}$,

$v=\left\{\begin{array}{l}\left(\frac{268}{\operatorname{CV} \mu^{0.50}}\right)^{1.96} \text { for tightly clustered population } \\ \left(\frac{250}{\operatorname{CV} \mu^{0.47}}\right)^{2.00} \text { for loosely clustered population }\end{array}\right.$

where $\mu$ is density $\left(\mathrm{m}^{-2}\right)$ and $\nu$ is sample size.

- If reduction of distance travelled is important, then twostage sampling is recommended. As an initial guide, half or less of the primary units should be selected and the number of quadrats (as determined by Equation 4) should be allocated among the selected primary units. Selection of quadrats within primary units should be by systematic sampling or similar logistically feasible design that provides good spatial balance.

- If it is desirable to maximize the collection and measurement of the target species of mussels for analyses of demographics or habitat relationships, then adaptive sampling designs should be considered. It should be noted that analyses and modelling should account for sampling probabilities for any unequal probability design. Unequal

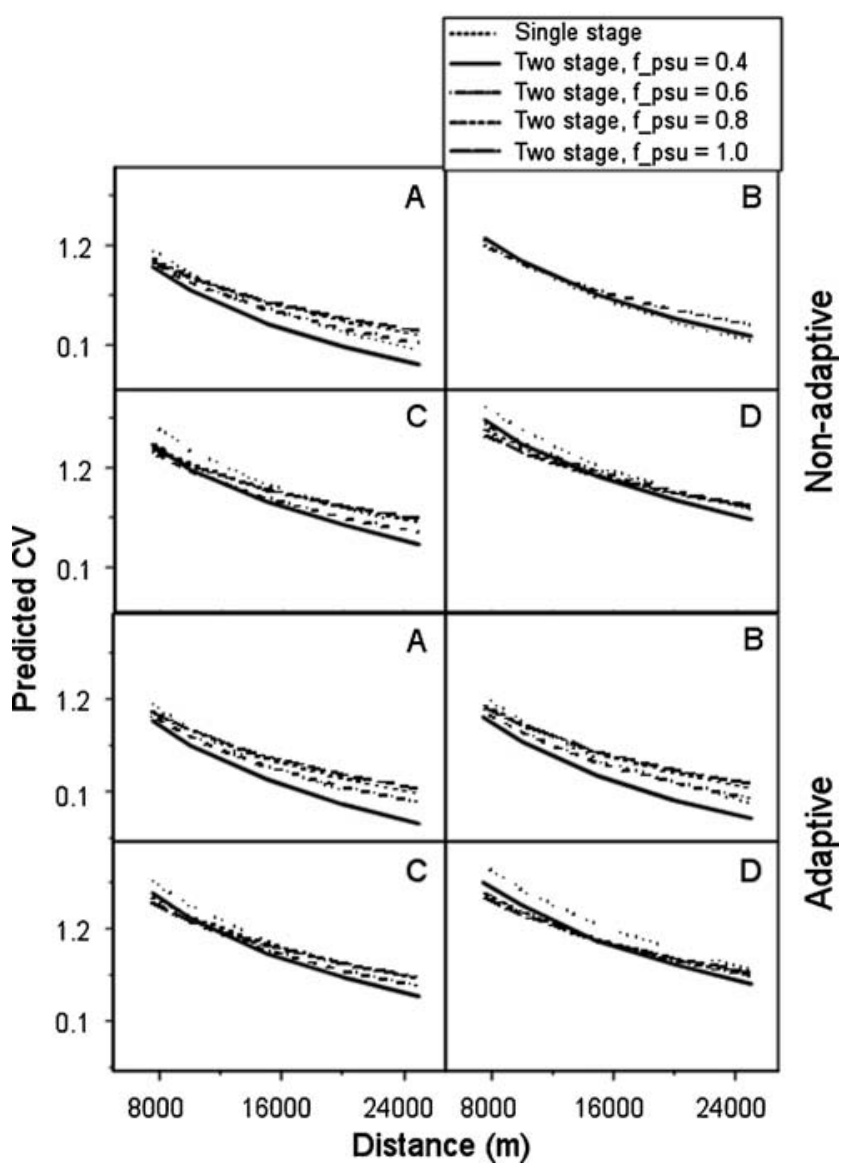

Figure 9. Predicted coefficient of variation (CV) as a function of spatial distribution, density, sampling design and distance travelled among sampling units. Distance travelled is a surrogate measure of sampling costs. Results from populations with loosely clustered spatial distributions (Matern process) are shown in panels $\mathrm{A}$ and $\mathrm{C}$, and those from populations with tightly clustered spatial distributions (Poisson cluster process) are shown in panels $\mathrm{B}$ and $\mathrm{D}$. Densities were $0.01 \mathrm{~m}^{-2}$ (panels A and B) and $0.001 \mathrm{~m}^{-2}$ (panels C and D). Conventional (non-adaptive) designs are in the top four panels, and adaptive designs are in the bottom four panels. For two-stage designs, there were a total of 20 primary units for all cases; the fraction of these that were sampled (i.e. $f_{\text {psu }}$ ) varied from 0.4 to 1.0

sampling probabilities are characteristic of many designs. Adaptive sampling is an unequal probability design, as are stratified sampling and model-based sampling designs.

\section{ACKNOWLEDGEMENTS}

This research was funded by the US Army Corps of Engineers, Environmental Management Program. The authors thank Doug Nichols for programming support, Mary Mandt for GIS support and Priscilla Young for database management. Penelope Pooler and Roger Haro provided helpful reviews of earlier drafts. 


\section{REFERENCES}

Agresti A. 1990. Categorical Data Analysis. Wiley: NY.

Brown JA, Manly BJF. 1998. Restricted adaptive cluster sampling. Environmental and Ecological Statistics 5: 49-63.

Brown JA. 2003. Designing an efficient adaptive cluster sample. Environmental and Ecological Statistics 10: 43-60.

Christman MC. 2000. A review of quadrat-based sampling or rare, geographically clustered populations. Journal of Agricultural, Biological, and Environmental Statistics 5: 168-201.

Diggle PJ. 1983. Statistical Analysis of Spatial Point Patterns. Academic Press: New York.

Dorazio RM. 1999. Design-based and model-based inference in surveys of freshwater mollusks. Journal of the North American Benthological Society 18: 118-131.

Downing JA, Downing WL. 1992. Spatial aggregation, precon, and power of surveys of freshwater mussel populations. Canadian Journal of Fisheries and Aquatic Sciences 49: 985-991.

Holland-Bartels LE. 1990. Physical factors and their influence on the mussel fauna of a main channel border habitat of the upper Mississippi River. Journal of the North American Benthological Society 9: 327-335.

Hosmer DW, Lemeshow S. 1989. Applied Logistic Regression. Wiley: NY.

Morales Y, Weber LJ, Weber, Mynett AE, Newton TJ. 2006. Effects of substrate and hydrodynamic conditions on the formation of mussel beds in a large river. Journal of the North American Benthological Society 25: 664-676.

Morrison LW, Smith DR, Nichols DW, Young CC. 2008. Using computer simulations to evaluate sample design: an example with the Missouri bladderpod. Population Ecology 50: 417-425.

Petitgas P. 2003. A method for the identification and characterization of clusters of schools along the transect lines of fisheries-acoustic surveys. ICES Journal of Marine Science 60: 872-884.

Pooler PS, Smith DR. 2005. Optimal sampling design for estimating spatial distribution and abundance of a freshwater mussel population. Journal of North American Benthological Society 24: 525-537.

Ricciardi A, Rasmussen JB. 1999. Extinction rates of North American freshwater fauna. Conservation Biology 13: 1220-1222.

Salehi MM, Seber GAF. 1997. Two-stage adaptive cluster sampling. Biometrics 53: 959-970.
Salehi MM, Smith DR. 2005. Two-stage sequential sampling: a neighborhood-free adaptive sampling procedure. Journal of Agricultural, Biological, and Environmental Statistics 10: 84-103.

Smith DR, Brown JA, Lo NCH. 2004. Application of adaptive cluster sampling to biological populations. In Sampling Rare or Elusive Species: Concepts, Designs, and Techniques for Estimating Population Parameters, Thompson WL (ed.). Island Press: Covelo, CA; 77122.

Smith DR, Villella RF, Lemarié DP. 2001a. Survey protocol for assessment of endangered freshwater mussels in the Allegheny River, Pennsylvania. Journal of North American Benthological Society 20: 118132

Smith DR, Villella RF, Lemarié DP, von Oettingen S. 2001b. How much excavation is needed to monitor freshwater mussels? In Freshwater Mollusk Symposium Proceedings, Tankersley RA, Warmolts DI, Watters GT, Armitage BJ, Johnson PD, Butler RS (eds). Ohio Biological Survey: Columbus, Ohio; 203-218.

Smith DR, Villella RF, Lemarié DP. 2003. Application of adaptive cluster sampling to low-density populations of freshwater mussels. Environmental and Ecological Statistics 10: 7-15.

Strayer DL, Downing JA, Haag WR, King TL, Layzer JB, Newton TJ, Nichols SJ. 2004. Changing perspectives on pearly mussels, North America's most imperiled animals. BioScience 54: 429-439.

Strayer DL, Smith DR. 2003. A guide to sampling freshwater mussel populations. American Fisheries Society Monograph No. 8, Bethesda, MD.

Thompson SK. 2002. Sampling. Wiley: New York.

Thompson SK, Seber GAF. 1994. Detectability in conventional and adaptive sampling. Biometrics 50: 712-724.

Thompson SK, Seber GAF. 1996. Adaptive Sampling. Wiley: New York.

Upper Mississippi River Conservation Committee (UMRCC). 1990. A strategic plan for the management of the freshwater mussel resource of the Upper Mississippi River. Upper Mississippi River Conservation Committee: Rock Island, IL.

Villella RF, Smith DR. 2005. Two-phase sampling to estimate river-wide populations of freshwater mussels. Journal of North American Benthological Society 24: 357-368. 